\title{
The Bactericidal Activity of Moxifloxacin in Patients with Pulmonary Tuberculosis
}

\author{
Roly D. Gosling, Leonard O. Uiso, Noel E. Sam, Emily Bongard, Esther G. Kanduma, Mramba Nyindo, \\ Richard W. Morris, and Stephen H. Gillespie \\ Departments of Medical Microbiology and Primary Care and Population Sciences, Royal Free and University College London Medical Schools, \\ Royal Free Campus, London, United Kingdom; Kibong'oto National Tuberculosis and Leprosy Hospital, Sanya Juu; and Clinical Laboratory, \\ Kilimanjaro Christian Medical College, Moshi, Tanzania
}

\begin{abstract}
Patients in whom acid-fast bacilli smear-positive pulmonary tuberculosis was newly diagnosed were randomized to receive $400 \mathrm{mg}$ moxifloxacin, $300 \mathrm{mg}$ isonaizid, or $600 \mathrm{mg}$ rifampin daily for 5 days. Sixteen-hour overnight sputa collections were made for the 2 days before and for 5 days of monotherapy. Bactericidal activity was estimated by the time taken to kill $50 \%$ of viable bacilli $\left(v t_{50}\right)$ and the fall in sputum viable count during the first 2 days designated as the early bactericidal activity (EBA). The mean vt ${ }_{50}$ of moxifloxacin was 0.88 days ( $95 \%$ confidence interval $[\mathrm{Cl}], 0.43-1.33$ days) and the mean EBA was $0.53(95 \% \mathrm{Cl} 0.28-0.79)$. For the isoniazid group, the mean vt $\mathrm{t}_{50}$ was 0.46 days $(95 \% \mathrm{Cl}, 0.31-0.61$ days) and the mean EBA was 0.77 (95\% Cl, 0.54-1.00). For rifampin, the mean vt $\mathrm{vt}_{50}$ was 0.71 days $(95 \% \mathrm{Cl}, 0.48-0.95$ days) and the mean EBA was 0.28 (95\% Cl, 0.15-0.41). Using the EBA method, isoniazid was significantly more active than rifampin $(p<0.01)$ but not moxifloxacin. Using the $v_{50}$ method, isoniazid was more active than both rifampin and moxifloxacin $(p=0.03)$. Moxifloxacin has an activity similar to rifampin in human subjects with pulmonary tuberculosis, suggesting that it should undergo further assessment as part of a short course regimen for the treatment of drug-susceptible tuberculosis.
\end{abstract}

Keywords: clinical trials; Africa; tuberculosis; quinolone

The continuing increase in tuberculosis infection and mortality has prompted researchers to seek new drugs to improve treatment (1). Since the introduction of rifampin and the rediscovery of the place of pyrazinamide in therapy, there has been no improvement in tuberculosis therapeutic regimens $(2,3)$. In addition, the prevalence of multiple drug resistance is rising worldwide, providing another stimulus to drug development $(1,4)$.

The fluorquinolones have been studied for their therapeutic potential in the management of tuberculosis (5). These bactericidal drugs, which inhibit DNA gyrase, are highly active against Mycobacterium tuberculosis, including strains resistant to firstline drugs $(6,7)$. The excellent in vitro activity is confirmed by bactericidal activity in a mouse model (8). Moxifloxacin has been shown to inhibit the growth of the main species of mycobacteria infecting humans $(7,9)$. Studies of the activity of moxifloxacin in mouse models also suggest that it has important bactericidal activity $(10,11)$.

Early phase studies of ciprofloxacin and a controlled clinical trial demonstrate useful bactericidal activity in humans (12-14). Patients with tuberculosis who were treated with rifampin, isoni-

(Received in original form May 22, 2003; accepted in final form August 11, 2003) Supported by Bayer AG.

Correspondence and requests for reprints should be addressed to Stephen $\mathrm{H}$. Gillespie, M.D., FRCP(Edin), FRCPath, Department of Medical Microbiology, Royal Free and University London College Medical Schools, Royal Free Campus, Rowland Hill Street, London NW3 2PF, UK. E-mail: stepheng@rfc.ucl.ac.uk

Am J Respir Crit Care Med Vol 168. pp 1342-1345, 2003

Originally Published in Press as DOI: 10.1164/rccm.200305-6820C on August 13, 2003

Internet address: www.atsjournals.org azid, and ciprofloxacin remained culture-positive for a more prolonged period than patients treated with a standard fourdrug regimen, and there was a trend toward a higher relapse rate among patients receiving ciprofloxacin, suggesting that this regimen had inferior sterilizing ability (12). A recent study demonstrated that high cure rates could be obtained with regimens of 4 to 5 months, which contained ofloxacin for the first 3 months (15). Quinolones are now used in patients who are not able to tolerate first-line agents or who are infected with multiple drug-resistant strains (16).

Bactericidal activity of antituberculosis antibiotics is important early in the course of treatment by ensuring a rapid reduction in the infective load and later in killing semidormant organisms to sterilize the lesions $(17,18)$. The first of these characteristics is tested in monotherapy studies also called early bactericidal activity (EBA) studies. These Phase II trials form an important part of the development of new antituberculosis drug $(19,20)$, giving confirmation that the activity demonstrated in vitro and in animal studies is translated into human subjects. It is also possible to compare the relative activity of the drugs with control regimens examined at the same time and with historic controls. In this paper, the results of a trial comparing moxifloxacin with rifampin and isoniazid are reported.

\section{METHODS}

\section{Study Location}

Patients were recruited from those presenting to the Kibong'oto National Tuberculosis Hospital, Sanya Juu, Tanzania with acid-fast bacilli smear-positive pulmonary tuberculosis.

\section{Ethical Considerations}

The study was approved by the ethical committee of the Kilimanjaro Christian Medical College, the National Institute of Medical Research, and the National Aids Control Program of Tanzania. Each patient gave witnessed oral consent.

\section{Patients}

Inclusion criteria for the study were (1) presence of acid alcohol-fast organisms found in a Ziehl-Neelsen-stained smear of sputum, (2) mild to moderate disease on clinical grounds, (3) age over 18, (4) no prior chemotherapy, (5) production of an adequate volume of sputum, (6) weight between 40 and $60 \mathrm{~kg}$, (7) consent to human immunodeficiency virus (HIV) serology. Patients were excluded from the study if they had severe or rapidly progressive disease or had any serious concomitant condition or renal or hepatic failure as judged by the admitting physician. Patients were also excluded if there was a history of hypersensitivity to any of the trial agents.

\section{HIV Serology}

After counseling and informed consent, patients serum was collected for an HIV test. The presence of HIV 1 and 2 antibodies were detected using two Enzyme immunoassays: Murex 1.2.0 (Murex Biotech Limited, Dartford, UK) and Vironostika, HIV Uni-Form IIplus O (Organon Tek- 
nika bv, Boxtel, Netherlands). Patients were considered to be HIVpositive only if both tests gave a positive result.

\section{Regimens Employed}

Patients were randomized by lot to receive one of the following regimens: isoniazid, $300 \mathrm{mg}$ daily; rifampin, $600 \mathrm{mg}$ daily; or moxifloxacin, $400 \mathrm{mg}$ daily for 5 days. Patients had two sputum collections before therapy was started. After completion of 5 days of monotherapy, patients were commenced on standard chemotherapy consisting of rifampin, isoniazid, pyrazinamide, and ethambutol in the intensive phase.

\section{Measuring Sputum Viable Count}

Sputum collection commenced from 4:00 P.M. on Day 0 (the day of admission) to 8:00 A.M. the next morning and continued daily during the duration of treatment, i.e., two pretreatment collections and a collection for each day of treatment. Sputum was collected in $200 \mathrm{ml}$, widemouthed, screw-capped, plastic "honey pots." The specimens were transported on ice each morning to the Kilimanjaro Christian Medical College for processing.

\section{Bacteriologic Procedures}

Sputum viable count was measured as follows. The specimen was homogenized by adding a Teflon-coated follower bar and stirring magnetically for 30 minutes. Five milliliters of the homogenized sputum was transferred to a 20-ml sterile universal plastic bottle, and an equal volume of 1:10 dithiothreitol (Sputasol; Oxoid, Basingstoke, UK) was added to produce a final ratio of 1 (dithiothreitol) to 1 (sputum). The sample was vortex-mixed for 20 seconds and then shaken mechanically for 15 to 20 minutes. The digested sputum samples were used to prepare a dilution series of: neat, $1 \times 10^{-1}, 1 \times 10^{-2}, 1 \times 10^{-3}, 1 \times 10^{-4}$, and $1 \times 10^{-5}$ made up in $0.2 \%$ bovine albumin. An aliquot of $100 \mu$ of the neat sputum:sputasol admix was inoculated in duplicate onto $7 \mathrm{H} 11$ Middlebrook agar made selective by the addition of Polymyxin B sulfate 200 units/ml, Carbenicillin (100 mg/L), Trimethoprim lactate $(20 \mathrm{mg} / \mathrm{L})$, Amphotericin B (100 mg/L), using Selectatabs (Mast, Bootle, UK). A series of $50 \mu \mathrm{l}$ aliquots of the dilutions were made on between 4 and 6 duplicate plates onto selective 7H11 Middlebrook medium. The plates were incubated in a humidified atmosphere for 3 weeks. The number of colonies was read on each plate, and the sputum viable count was calculated using the dilution that provided a count of less than 80 colonies. Drug susceptibility testing was not performed on the isolates obtained in this study.

\section{Calculation of Bactericidal Activity}

Time to reduce viable bacilli count by $50 \%$. The results of sputum viable count (the pretreatment sputa colony count was calculated as the mean of the two pretreatment samples) were fitted to a single phase exponential decay curve as described previously using the equation $\mathrm{V}=$ $\mathrm{Me}^{-\mathrm{kt}}+\mathrm{S}$, where $\mathrm{V}$ is the viable count, $\mathrm{M}$ is the number of bacteria susceptible to antibiotic action, e is the Napierian constant, $\mathrm{k}$ is the exponential decay constant, $\mathrm{t}$ is the time, and $\mathrm{S}$ is the plateau reached at the end of the study (17). Curve fitting was performed using the GraphPad Prism program (GraphPad Software, San Diego, CA). Results were only accepted if the goodness of fit, measured by a calculation of $r^{2}$, was greater than 0.95 . Using values of $\mathrm{k}$ derived from the curve fitting, it was possible to calculate the time (in days) taken to reduce the viable count by $50 \%\left(\mathrm{vt}_{50}\right)$ using the formula $\mathrm{vt}_{50}=\ln (0.5) / \mathrm{k}(17)$. When curves failed to fit the model, discrepant values were removed using the rule base described previously (17). Briefly, the single point was removed sequentially and taking the remaining points a new value for $r^{2}$ was recalculated. All possible combinations of data were then calculated, and the best fit was compared. If only one change brought the $r^{2}$ to above 0.95 , that result was accepted, but if more than one of the deletions brought a value of $r^{2}$ above 0.95 , the data set with the lowest variance was accepted as the best estimate and was included in further calculations. If removal of a single point was insufficient, then an additional point was removed provided that at least four points were available. The results of the recalculation were treated as mentioned previously.

EBA calculation. Measurement of EBA depends on calculating half of the ratio between the Day 0 (the mean of both pretreatment sputa collections) and Day 2 viable counts expressed as a log using the equation: $\mathrm{EBA}=\left(\log _{10} \mathrm{cfu} / \mathrm{ml} \mathrm{S}_{0}-\log _{10} \mathrm{cfu} / \mathrm{ml} \mathrm{S}_{2}\right) / 2$, where $\mathrm{S}_{0}$ and $\mathrm{S}_{2}$ are the initial (mean of the two pretreatment samples) and Day 2 colony counts, respectively (18).

\section{Statistical Tests}

Data were entered into an Excel spreadsheet. Curve fitting was done using GraphPad Prism. All other statistical tests were performed using SPSS 9.0.

\section{RESULTS}

A total of 43 patients with smear-positive pulmonary tuberculosis, of whom 37 (86\%) were male, were enrolled into the study and randomized to receive therapy as follows: isoniazid, 16 (one HIV-positive); moxifloxacin, 13 (three HIV-positive); and rifampin, 14 (one HIV-positive). The only significant difference in demographics between the groups was that the mean weight of patients in the isoniazid arm was significantly lower than both moxifloxacin and rifampin arms. These data are described in full in Table 1.

\section{Calculation of Bactericidal Activity}

Time to reduce viable count $50 \%$. In the moxifloxacin group, the data of 8 out of 13 subjects fitted the model without adjustment and that of 3 subjects fitted it further with reiteration. A total of two subjects were excluded because they had less than four data points making it impossible to estimate $\mathrm{vt}_{50}$ reliably. An example of the decline in sputum viable count for a patient treated with moxifloxacin is illustrated in Figure 1A. Out of 16 subjects in the isoniazid group, the data of 11 subjects fitted the exponential decay model without adjustment and that of 2 fitted the model with reiteration. Three patients were excluded because they had less than four data points. An example of the decline in sputum viable count in a patient treated with isoniazid is illustrated in Figure 1B. Within the rifampin group, the data of 4 out of 14 subjects fitted the model without adjustment and that of 4 subjects fitted it with reiteration. A total of 6 subjects were excluded because they had less than four data points. An example of the decline in sputum viable count for patients treated with rifampin is illustrated in Figure 1C. The mean values for $\mathrm{vt}_{50}$ are recorded in Table 2. Using the Kruskal-Wallis analysis of variance, we showed that isoniazid group had a higher bactericidal activity than both rifampin and moxifloxacin groups (asymptotic $\mathrm{p}=0.03$ ).

TABLE 1. DEMOGRAPHIC DATA OF PATIENTS IN TRIAL

\begin{tabular}{|c|c|c|c|c|c|}
\hline Variable & $\begin{array}{l}\text { Isoniazid Group } \\
\quad(\mathrm{n}=16)\end{array}$ & $\begin{array}{l}\text { Moxifloxacin Group } \\
\qquad(\mathrm{n}=13)\end{array}$ & $\begin{array}{l}\text { Rifampin Group } \\
\quad(\mathrm{n}=14)\end{array}$ & Test & p Value \\
\hline Male (\%) & $13(81)$ & $11(85)$ & $13(93)$ & $\chi^{2}$ test & 0.65 \\
\hline HIV-positive (\%) & $1(6)$ & $3(23)$ & $1(7)$ & $x^{2}$ test & 0.31 \\
\hline Median age, yr (range) & $33(20-54)$ & $32(21-60)$ & $28.5(18-70)$ & Kruskal-Wallis test & 0.4 \\
\hline Mean weight, kg (SD) & $48.6(4.7)$ & $52.8(4.4)$ & $53.9(5.2)$ & $t$ test & 0.01 \\
\hline
\end{tabular}

Definition of abbreviation: HIV = human immunodeficiency virus. 
A

Patient 12

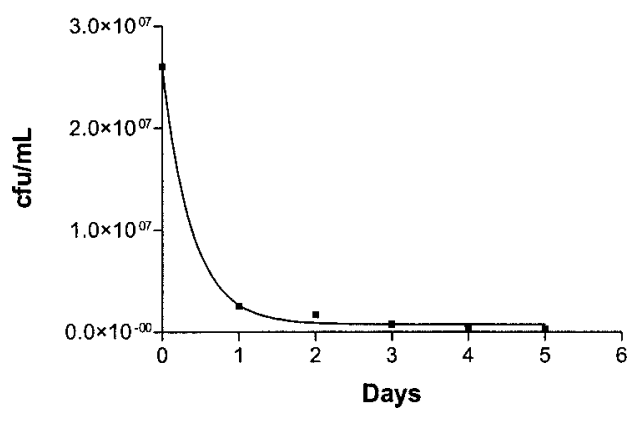

B

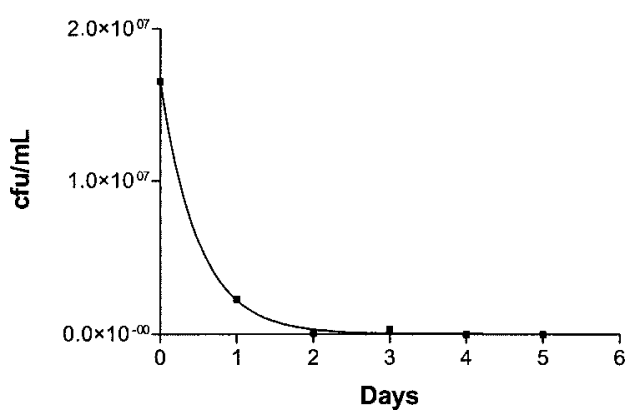

C

Patient 16

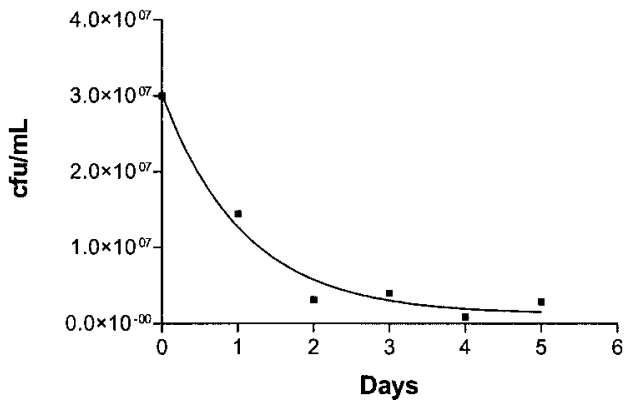

Figure 1. (A) Graph illustrating the fall in sputum viable count (cfu/ml) for a patient treated with isoniazid. (B) Graph illustrating the fall in sputum viable count $(\mathrm{cfu} / \mathrm{ml})$ for a patient treated with moxifloxacin. (C) Graph illustrating the fall in sputum viable count (cfu/ml) for a patient treated with rifampin.

EBA calculation. In the moxifloxacin group, 8 out of 13 subjects had complete data for Day 0 and Day 2 (five subjects were excluded as they had missing data for Day 0 and/or Day 2). In the isoniazid group, 12 out of 16 subjects were included in the

TABLE 2. MEAN TIME TO KILL $50 \%$ OF VIABLE BACILLI FOR ISONIAZID, MOXIFLOXACIN, AND RIFAMPIN GROUPS

\begin{tabular}{lrcccc}
\hline & & & & \multicolumn{2}{c}{$95 \%$ Confidence Interval for Mean } \\
\cline { 5 - 6 } Drug & $\mathrm{n}$ & Mean vt $_{50}(d)$ & $\mathrm{SD}$ & Lower Bound & Upper Bound \\
\hline Moxifloxacin & 11 & 0.88 & 0.67 & 0.43 & 1.33 \\
Isoniazid & 14 & $0.46^{*}$ & 0.26 & 0.31 & 0.61 \\
Rifampin & 8 & 0.71 & 0.32 & 0.48 & 0.95 \\
Total & 33 & & & & \\
& & & & &
\end{tabular}

Definition of abbreviation: $\mathrm{vt}_{50}=$ time to kill $50 \%$ of viable bacilli.

* Mean $\mathrm{vt}_{50}$ of isoniazid is significantly different from both moxifloxacin and rifampin (asymptotic $p=0.03$ ) using the Kruskal-Wallis analysis of variance. analysis of EBA in the isoniazid group. In all, 12 out of 14 subjects in the rifampin group had complete data for Day 0 and Day 2. The mean EBA values are recorded in Table 3. In contrast to the $\mathrm{vt}_{50}$ results, a Kruskal-Wallis analysis of variance suggested that the isoniazid EBA was significantly greater than that of rifampin (asymptotic $\mathrm{p}=0.006$ ), but there was no significant difference in the EBA of moxifloxacin and both isoniazid and rifampin.

Contamination of culture plates with environmental fungi was responsible for all missing data points except in the case of three subjects (two subjects treated with rifampin, both HIV negative, and one subject treated with moxifloxacin, HIV-positive) where $M$. tuberculosis did not grow on the $7 \mathrm{H} 11$ media.

\section{DISCUSSION}

There are many major challenges facing clinicians working to meet the global pandemic of tuberculosis. The first is to identify agents to treat drug-resistant tuberculosis. Others include developing regimens that shorten the length of therapy and treating latent disease $(1,19)$.

Monotherapy studies provide the opportunity to assess the bactericidal activity of new agents in human subjects (some of the data presented in this paper was presented at a "Work in Progress" meeting of the Royal Society of Tropical Medicine and Hygiene, 2003 [21]). All previous studies have demonstrated that isoniazid is the most bactericidal agent in current use. In contrast, rifampin, which is also central to effective chemotherapy, has only a modest activity in studies that have measured the bactericidal activity over 2 days $(18,22)$. Our group believes that this period is too short to measure activity and advocated a minimum of 5-day studies (17). Our results confirm prior studies showing that isoniazid is highly bactericidal (our EBA $=0.46$ and $\mathrm{SD}=0.26$, compared with prior EBAs of $0.43-0.72$ and SDs $0.17-0.54)(17,18,23-25)$. Our results for rifampin $(\mathrm{EBA}=$ $0.28, \mathrm{SD}=0.71)$ are similar to results published elsewhere $(0.19$ [18], 0.29, SD =0.31 [23], and 0.20, SD =0.04 [24]).

This paper confirms that moxifloxacin's activity, demonstrated in vitro and in mouse models (7-11), extends to humans. For moxifloxacin, the time to reduce the viable count by $50 \%$ $\left(\mathrm{vt}_{50}\right)$ is 0.88 days $(95 \%$ confidence interval, $0.43-1.33$ ), and when calculated by the EBA methodology for bactericidal activity the mean $\log$ fall in sputum viable count is 0.53 (95\% confidence interval, $0.28-0.79)$. The activity of moxifloxacin is similar to that demonstrated for rifampin when calculated by both methods.

From our results, the EBA of moxifloxacin (0.53) is higher than that of ciprofloxacin $(0.20-0.21[12,26])$ and ofloxacin $(0.30)$ (25). However, the $\mathrm{vt}_{50}$ for ciprofloxacin, $750 \mathrm{mg}$ daily was similar to that of moxifloxacin ( 0.79 and 0.88 , respectively). We should be cautious in comparing our data with other quinolones tested

TABLE 3. MEAN EARLY BACTERICIDAL ACTIVITY OF ISONIAZID, MOXIFLOXACIN, AND RIFAMPIN GROUPS

\begin{tabular}{lrcccc}
\hline & & & & \multicolumn{2}{c}{$95 \% \mathrm{Cl}$ for Mean } \\
\cline { 5 - 6 } Drug & $\mathrm{n}$ & Mean EBA & SD & Lower Bound & Upper Bound \\
\hline Moxifloxacin & 8 & 0.53 & 0.31 & 0.28 & 0.79 \\
Isoniazid & 12 & 0.77 & 0.37 & 0.54 & 1.00 \\
Rifampin & 12 & $0.28^{*}$ & 0.21 & 0.15 & 0.41 \\
Total & 32 & & & & \\
\hline
\end{tabular}

Definition of abbreviations: $\mathrm{Cl}=$ confidence interval; $\mathrm{EBA}=$ early bactericidal activity.

* Mean EBA of rifampin is significantly lower than that of isoniazid (asymptotic $\mathrm{p}<0.01$ ) using the Kruskal-Wallis analysis of variance. 
previously due to differences in methodology and small patient groups.

It is notable that using the $\mathrm{vt}_{50}$ methodology we detected a significant difference between isoniazid and both rifampin and moxifloxacin. In contrast, using the EBA method we demonstrated that rifampin alone has significantly less activity than isoniazid and that there is no difference between moxifloxacin and isoniazid. The reasons for these different results are not clear. Evaluation of larger groups of patients may help detect a significant difference between the EBA of isoniazid and moxifloxacin. There is some debate over the modeling of EBA studies, but there is general acceptance that exponential decay curves correctly model the fall in viable bacilli in the early part of treatment in the patient population used in these studies (27).

There are several limitations in this study. In the design of the study, we planned to recruit at least five HIV-seropositive subjects in each group However, our inclusion criteria of patients having mild to moderate smear-positive pulmonary tuberculosis led to the exclusion of most patients who were HIV-positive. Consequently, it was impossible to draw any specific conclusions to the activity of these agents in patients infected with HIV. In the laboratory, we had high rates of fungal contamination, which resulted in the loss of 25/215 (11.6\%) data points and eight and seven patients being excluded from the EBA and $\mathrm{vt}_{50}$ calculations, respectively. Drug susceptibility testing was not performed and this may have been a limitation, but resistance to antituberculosis agents is low at our study site and previous studies detected no quinolone-resistant M. tuberculosis (14).

The data reported in this paper confirms that the excellent in vitro activity of moxifloxacin translates into activity in the human host. Moxifloxacin joins the small number of highly bactericidal antituberculosis drugs and may contribute to the development of more effective regimens. Further clinical studies are needed to determine whether the moxifloxacin adds to the bactericidal activity of isoniazid or rifampin. Clinical trials to determine whether regimens containing moxifloxacin bring higher rates of culture conversion at 2 months should be performed as soon as sufficient safety data are available.

Conflict of Interest Statement: R.D.G. study and salary was supported by a grant from Bayer AG; L.O.U. has no declared conflict of interest; N.E.S. has no declared conflict of interest; E.B. has no declared conflict of interest; E.G.K. has no declared conflict of interest; M.N. has no declared conflict of interest; R.W.M. has no declared conflict of interest; S.G.G. received a grant from Bayer AG to perform this study, and has additionally given lectures at the request of Bayer AG during the period of the study.

Acknowledgment: The authors are grateful for the technical support provided by A. Ole Solulu, E. Ngomou, G. Kisyombe, and all the staff at Kibong'oto Hospital.

\section{References}

1. Pablos-Mendez A. Working alliance for TB drug development, Cape Town, South Africa, February 8th, 2000. Int J Tuberc Lung Dis 2000;4: 489-490.

2. Fox W, Ellard GA, Mitchison DA. Studies on the treatment of tuberculosis undertaken by the British Medical Research Council tuberculosis units, 1946-1986, with relevant subsequent publications. Int J Tuberc Lung Dis 1999;3:S231-S279.

3. Fox W. Whither short-course chemotherapy? Br J Dis Chest 1981;75: 331-357.

4. Espinal MA, Laszlo A, Simonsen L, Boulahbal F, Kim SJ, Reniero A, Hoffner S, Rieder HL, Binkin N, Dye C, et al. Global trends in resistance to antituberculosis drugs: World Health Organization: International Union against Tuberculosis and Lung Disease Working Group on Anti-Tuberculosis Drug Resistance Surveillance. N Engl J Med 2001; 344:1294-1303.
5. Gillespie SH, Kennedy N. Fluoroquinolones: a new treatment for tuberculosis? Int J Tuberc Lung Dis 1998;2:265-271.

6. Drlica K, Zhao X. DNA gyrase, topoisomerase IV, and the 4-quinolones. Microbiol Mol Biol Rev 1997;61:377-392.

7. Gillespie SH, Billington O. Activity of moxifloxacin against mycobacteria. J Antimicrob Chemother 1999;44:393-395.

8. Ji B, Lounis $\mathrm{N}$, Truffot-Pernot $\mathrm{C}$, Grosset J. In vitro and in vivo activities of levofloxacin against Mycobacterium tuberculosis. Antimicrob Agents Chemother 1995;39:1341-1344.

9. Rodriguez JC, Ruiz M, Climent A, Royo G. In vitro activity of four fluoroquinolones against Mycobacterium tuberculosis. Int J Antimicrob Agents 2001;17:229-231.

10. Ji B, Lounis N, Maslo C, Truffot-Pernot C, Bonnafous P, Grosset J. In vitro and in vivo activities of moxifloxacin and clinafloxacin against Mycobacterium tuberculosis. Antimicrob Agents Chemother 1998;42: 2066-2069.

11. Miyazaki E, Miyazaki M, Chen JM, Chaisson RE, Bishai WR. Moxifloxacin (BAY12-8039), a new 8-methoxyquinolone, is active in a mouse model of tuberculosis. Antimicrob Agents Chemother 1999;43:85-89.

12. Kennedy N, Fox R, Kisyombe GM, Saruni AO, Uiso LO, Ramsay AR, Ngowi FI, Gillespie SH. Early bactericidal and sterilizing activities of ciprofloxacin in pulmonary tuberculosis. Am Rev Respir Dis 1993;148: 1547-1551.

13. Kennedy N, Fox R, Uiso L, Ngowi FI, Gillespie SH. Safety profile of ciprofloxacin during long-term therapy for pulmonary tuberculosis. J Antimicrob Chemother 1993;32:897-902.

14. Kennedy N, Berger L, Curram J, Fox R, Gutmann J, Kisyombe GM, Ngowi FI, Ramsay AR, Saruni AO, Sam N, et al. Randomized controlled trial of a drug regimen that includes ciprofloxacin for the treatment of pulmonary tuberculosis. Clin Infect Dis 1996;22:827-833.

15. Tuberculosis Research Centre. Shortening short course chemotherapy: a randomised clinical trial for treatment of smear-positive pulmonary tuberculosis with regimens using ofloxacin in the intensive phase. Ind J Tuberc 2002;49:27-38.

16. Yew WW, Lee J, Wong PC, Kwan SY. Tolerance of ofloxacin in the treatment of pulmonary tuberculosis in presence of hepatic dysfunction. Int J Clin Pharmacol Res 1992;12:173-178.

17. Gillespie SH, Gosling RD, Charalambous BM. A re-iterative method for calculating the early bactericidal activity of anti-tuberculosis drugs. Am J Respir Crit Care Med 2002;166:31-35.

18. Jindani A, Aber VR, Edwards EA, Mitchison DA. The early bactericidal activity of drugs in patients with pulmonary tuberculosis. Am Rev Respir Dis 1980;121:939-949.

19. O'Brien RJ. Scientific blueprint for tuberculosis drug development Tuberculosis 2001;81:19-45.

20. O'Brien RJ. Studies of the early bactericidal activity of new drugs for tuberculosis: a help or a hindrance to antituberculosis drug development? Am J Respir Crit Care Med 2002;166:3-4.

21. Gosling RD, Uiso LO, Sam NE, Gillespie SH. The early antimicrobial activity of moxifloxacin (8-methoxyquinolone) in the treatment of pulmonary tuberculosis [abstract]. Trans $R$ Soc Trop Hyg (In press).

22. Donald PR, Sirgel FA, Botha FJ, Seifart HI, Parkin DP, Vandenplas ML, Van de Wal BW, Maritz JS, Mitchison DA. The early bactericidal activity of isoniazid related to its dose size in pulmonary tuberculosis. Am J Respir Crit Care Med 1997;156:895-900.

23. Chan SL, Yew WW, Ma WK, Girling DJ, Aber VR, Felmingham D, Allen BW, Mitchison DA. The early bactericidal activity of rifabutin measured by sputum viable counts in Hong Kong patients with pulmonary tuberculosis. Tuber Lung Dis 1992;73:33-38.

24. Sirgel F, Botha FJ, Parkin DP, Van de Wal BW, Donald PR, Clar PK, Mitchison D. The early bactericidal activity of rifabutin in patients with pulmonary tuberculosis measured by sputum viable counts: a new method of drug assessment. J Antimicrob Chemother 1993;32:867-875.

25. Sirgel FA, Donald PR, Odhiambo J, Githui W, Umapathy KC, Paramasivan CN, Tam CM, Kam KM, Lam CW, Sole KM, et al. A multicentre study of the early bactericidal activity of anti-tuberculosis drugs. J Antimicrob Chemother 2000;45:859-870.

26. Sirgel F, Botha FJ, Parkin DP, Van de Wal BW, Donald PR, Mitchison D. The early bactericidal activity of ciprofloxacin in patients with pulmonary tuberculosis. Am J Respir Crit Care Med 1997;156:901-905.

27. Jindani A, Dore CJ, Mitchison DA. Bactericidal and sterilizing activities of antituberculous drugs during the first 14 days. Am J Respir Crit Care Med 2003;167:1348-1354. 